\title{
P. Lond. V 1731: \\ Aurelia Tsone, ¿un ejemplo de vida ascética?
}

\author{
María Jesús ALBARRÁN MARTÍNEZ
}

P. Lond. V 1731 (585 d.C.) es una más de las disputas familiares, legales y económicas, que forman parte del denominado Archivo de Patermucio. Aurelia Tsone, una asceta, después de mantener un conflicto económico con su madre, Aurelia Tapia, recibe una cantidad de dinero que reclamaba como propio. Tsone puede responder al modelo de ascetismo urbano, puesto que se trata de una religiosa que tiene relaciones sociales, familiares y económicas de manera independiente. Esto parece indicar que el modelo de ascetismo femenino urbano continúa llevándose a cabo a finales del siglo VI d.C. en Egipto.

\section{P. Lond. V 1731: Aurelia Tsone, an example of ascetic life?}

P. Lond. V1731 (585 A.D.) features yet another of the familial legal and financial disputes in the Patermouthis Archive. Aurelia Tsone, a nun, after carrying on an economic dispute with her mother, Aurelia Tapia, received a sum of money from her, which she claimed as her own. Tsone may represent a type of urban ascetic since she is a nun with social, family and economic independent relationships. This may indicate that the female urban asceticism model continues to exist in late sixth century Egypt.

KEY words: Syene, Byzantine Egypt, VIth century A.D., Patermouthis Archive, ascetism

$P$ Lond. V $1731^{1}$ pertenece al llamado "Archivo de Patermucio" 2 , que consta de cincuenta y tres papiros, la mayor parte escritos en lengua griega, de los que sólo nueve están en copto. Todos son en su mayoría transacciones económicas de la familia de Patermucio y de su mujer Kako. Los documentos proceden de Siene y se datan cronológicamente entre los años 493-613 d.C.
El papiro en el que nos vamos a centrar es un recibo de cobro de dinero, fechado el 20 de septiembre del 585 d.C. en Siene, la actual Asuán. Se trata de un recibo escrito en griego, la lengua oficial de la administración. En él, Aurelia Tsone declara la obtención de una cantidad de dinero, cuatro sólidos de oro, que significa el fin de una lucha, posiblemente judicial, llevada a cabo contra su madre, Aurelia Tapia.

1. Todos los papiros que aparecen en este trabajo están citados según sus abreviaturas de acuerdo a J. F. Oates et alii, Checklist of Editions of Greek and Latin Papyri, Ostraca and Tablets, 5th ed., BASP Suppl. 9, 2001, disponible en Internet, con actualizaciones frecuentes, en: http://scriptorium.lib.duke.edu/papyrus /texts/clist.html. Agradezco a Helena Gimeno su interés para que yo participase en el III Congreso Ibérico de Egiptología, a José Domingo Rodríguez Martín las indicaciones que me facilitó sobre legislación romana, y a Sofía Torallas y Margarita Vallejo sus sugerencias y comentarios para la mejora de este trabajo. No obstante, los errores que pueda contener son únicamente responsabilidad mía.

2. Rustafjaell, 1909: 3-5; Bell, 1913: 160-174; Farber y Porten, 1986: 81-98; Clackson, 1995: 97-116; Geens, 2005: 1-9. Todos los nombres propios de este trabajo se han transcrito según se encuentran los nombres originales en los documentos del Archivo.

Fecha de recepción: 15 de enero de 2007

Fecha de admisión: 15 de mayo de 2007 


\section{P. LOND. V 1731}

${ }^{1}$ En el cuarto año del reinado de nuestro más divino señor Flavio Tiberio Mauricio el eterno Augusto ${ }^{2}$ emperador y gran benefactor, y en el segundo año del consulado de nuestro mismo señor, ${ }^{3} 23$ de Thoth de la cuarta indicción, en Siene. ${ }^{4}$ Aurelia Tsone, hija de Menas, y cuya madre es Tapia, monja (monaché), originaria de Siene, ${ }^{5}$ llevando también un signatario quien escribe por ella debajo, y testigos ${ }^{6}$ quienes testifican debajo esta ${ }^{7}$ acta como autorizada, a Aurelia Tapia, hija de Tsíos y Mariam, ${ }^{8}$ actuando ella misma sin la autoridad de un hombre que gestione sus bienes personales, a mi propia madre, originaria de la dicha ${ }^{9}$ Siene, saludos. Puesto que pronto mi fallecido padre Menas, te entregó ${ }^{10}$ legalmente la aportación del matrimonio y después de mi nacimiento, cuando yo todavía era pequeña, ${ }^{11}$ por la energía del diablo y de Satán fuiste apartada de él; ${ }^{12}$ y mi mencionado padre Menas, te dio cuatro monedas de oro; ${ }^{13}$ y después que tuve la edad legal, me dirigí contra ti acusándote a causa ${ }^{14}$ de las dichas cuatro monedas, diciéndote que te habían sido dadas ${ }^{15}$ estrictamente para mi alimentación durante mi infancia. Porque como se ha dicho, yo fui alimentada ${ }^{16}$ por mi padre; después que yo fui devuelta por ti y tú te uniste a otro hombre, ${ }^{17}$ me dirigí contra ti buscando tomar esas (monedas); tú las disputaste diciendo ${ }^{18}$ que las dichas cuatro monedas eran por tu liberación y dote, y después de varias ${ }^{19}$ reclamaciones, réplicas y opiniones, la última decisión fue ${ }^{20}$ que yo debería recibir las dichas cuatro monedas, declarando públicamente devolverme ${ }^{21}$ lo que era antes de mi padre, y al recibirlo yo no iría contra ti, ${ }^{22}$ ni te acusaría a ti, ni a tus herederos, ni ahora ni en ninguna ocasión a través de nadie ${ }^{23}$ sobre ningún dinero. Por lo cual ésta que suscribe, libre de preocupaciones, ${ }^{24}$ acuerdo nunca más decirte nada a ti, ni después a tus herederos, ${ }^{25}$ ni maldecir, ni acusarte en juicio a causa del tema, ${ }^{26}$ y ni dar, ni tomar ninguna palabra dirigida contra ti, ${ }^{27} \mathrm{ni}$ a tus herederos y des- cendientes ${ }^{28}$ en alguna ocasión sobre el asunto causado [... reconocimiento del acuerdo]. ${ }^{34}$ Por nuestra seguridad firmamos este acuerdo, el cual es válido, legal y firme ${ }^{35}$ donde quiera que sea presentado y mostrado, ${ }^{36}$ con la suscripción de aquel quien firma por mi debajo la petición y opinión, ${ }^{37}$ y con la de aquellos quienes testifican y garantizan en persona, ${ }^{38} \mathrm{y}$ en respuesta a la cuestión formal hemos dado nuestro asentimiento. (Firmado) Yo Aurelia Tsone, hija de Menas y Tapia, ${ }^{39}$ monja (monaché), de Siene, he hecho el presente acuerdo y acepto ${ }^{40}$ todo lo expuesto. Yo Flavio Ioannes hijo de Abraamios, actuario del número ${ }^{41}$ de Siene, habiendo escrito por ella, porque es iletrada. ${ }^{42}$ Yo, Patermucio hijo de Sereno, el más humilde presbítero de Siene, testigo. ${ }^{43}$ Yo, Flavio Allamón hijo de Díos, soldado del número de Siene, testigo. ${ }^{44}$ Yo, Flavio Paeíon hijo de Díos, soldado del número de Siene, testigo. ${ }^{45}$ Yo, Isakos Taeionos archidiácono de la iglesia de Santa Maria de Siene, testigo. ${ }^{46}$ Yo, Papnucio Aionos, diácono de la Iglesia de Siene, testigo. ${ }^{47}$ Yo, Flavio Dióscoro hijo de Ioannes, soldado del número de Siene, testigo. ${ }^{48}$ Yo, Flavio Ermías hijo de Ioannes, soldado del número de Siene, testigo.

${ }^{49}$ Realizado por mí, Marco, hijo de Apa Díos.

Verso. ${ }^{50}$ Acta autorizada por Aurelia Tsone hija de Menas, monja (monaché) de Siene con relación a Tapia, ${ }^{51}$ su madre.

\section{Situación familiar}

Las dos protagonistas de esta historia son las mencionadas Tsone y su madre Tapia. Mientras que Tapia aparece en varios documentos del archivo $^{3}$, Tsone es nombrada únicamente en este recibo. En todas las ocasiones en las cuales se menciona su nombre en el documento es denominada como $\mu$ ova $\chi \eta^{4}$. Esta designación alude a un título monástico ${ }^{5}$, por lo tanto se trata de una religiosa.

3. P. Münch. I 1; P. Münch. I $6+$ P. Lond. V 1849; P. Münch. I 9; P. Münch. I 11 y P. Lond. V 1733.

4. P. Lond. V 1731,1 s. 4,39 y 50.

5. Morard, 1973: 332-411; Judge, 1977: 72-89; Choat, 2002: 5-23. 


\section{Cuadro genealógico familiar}

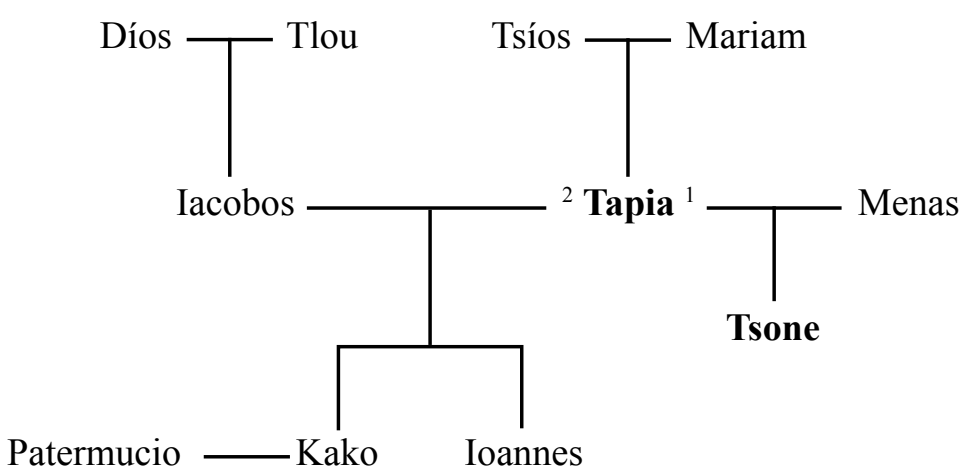

Tsone era hija de Menas y Tapia $^{6}$, los cuales se divorciaron cuando ella era muy pequeña ${ }^{7}$ y al parecer fue la única hija que tuvo en común el matrimonio.

De su madre, Tapia, sabemos que estuvo casada dos veces, primero con Menas, el padre de su hija Tsone, del cual no tenemos más datos, $\mathrm{y}$ en segundas nupcias con Iacobos ${ }^{8}$, un hombre de negocios que se dedicaba al transporte fluvial por el Nilo, quien había heredado el negocio familiar de su padre ${ }^{9}$. La familia de Iacobos, en la cual entró a pertenecer Tapia, era en cierta medida adinerada, y con una cierta posición social en la ciudad de Siene. Durante este segundo matrimonio, Tapia tuvo con Iacobos dos hijos, Ioannes y Kako, ésta última casada con Patermucio quien da nombre a este $\operatorname{archivo~}^{10}$. En este momento, en el año 585 d.C., Tapia había enviudado de su segundo marido, que había muerto dos años antes, en el 583 d. $\mathrm{C}^{11}$.

Tsone involucró a su madre en un proceso para reclamarle cuatro sólidos de oro, una suma de dinero al parecer destinada para la manutención durante su infancia ${ }^{12}$, que su padre, Menas, había entregado a su madre cuando ambos se divorciaron. Esto nos indica que la hija, tras el divorcio de sus padres, primero quedó bajo el cuidado de Tapia quien se hizo cargo de ella. Sin embargo, cuando la madre se casó por segunda vez no quiso quedarse con la niña ${ }^{13}$, y por eso fue el padre quien se hizo cargo de su tutela y cuidado ${ }^{14}$. Después de obtener la mayoría de edad-tras cumplir los doce años- es cuando Tsone decidió reclamar esos

6. P. Lond. V 1731, 1s. 4, 38 y 50.

7. P. Lond. V 1731, 1. 10.

8. Hijo de Díos y Tlou (Vid. cuadro genealógico familiar).

9. Iacobos es el único de sus tres hermanos que continúa con la profesión de su padre, Díos, y hereda el negocio familiar tras la muerte de éste; $P$. Münch. I 1.

10. Patermucio y Kako aparecen por primera vez como matrimonio en P. Lond. V 1724, fechado entre los años 578-582 d.C.

11. P. Lond. V $1849+$ P. Münch. I 6 ( ¿7 de junio? de 583 d.C.).

12. P. Lond. V 1731, 1. 15.

13. P. Lond. V 1731, 1. 16.

14. P. Lond. V 1731, 1s. $15-16$. 
cuatro sólidos de oro ${ }^{15}$. Según expone ella misma en el documento reclamó el dinero porque fue cuidada y alimentada por su padre. Esta suma de dinero correspondía a Menas haberla reclamado, y si es su hija quien lo reclamó, es bastante posible que Menas hubiese muerto ${ }^{16}$, y ella podría así solicitar este dinero como heredera única. Tapia disputó los cuatro sólidos con su hija alegando que le fueron entregados por su "liberación y dote" que la cantidad era la recuperación de su dote matrimonial con Menas, y quedaba libre de toda unión con él.

Ambas llevaron a cabo una disputa a causa de esta suma de dinero, mencionándose en el papiro, que se hubieron hecho reclamaciones y réplicas por ambas partes, hasta llegar a esta resolución ${ }^{18}$. El acuerdo final recayó a favor de Tsone, y Tapia tuvo que entregarle los cuatro sólidos de oro $^{19}$. Es entonces, en este papiro, donde Tsone hace una declaración pública de haber recibido el dinero de su madre, y se compromete a no reclamarle nada más, ni a ella ni a sus herederos y descendientes ${ }^{20}$.

Este documento nos ofrece algunos datos sobre la vida de esta familia. Sin embargo, para la reconstrucción de la historia, tienen que tenerse en cuenta otros documentos del archivo. Tsone, al ser hija de Tapia, está relacionada, aunque no sea directamente, con la familia del archivo; la reclamación que ella efectúa es por tanto, una más de las disputas familiares que se conservan en dicho $\operatorname{archivo}^{21}$.

En el documento Tapia está divorciada de un primer marido, Menas, y viuda del segundo, Iacobos, que había muerto dos años antes, en el 583 d.C., como indican P. Lond. V 1849 y P. Münch. I 6.

En el mismo momento de la lucha con Tsone, Tapia está involucrada en la repartición de los bienes de su marido entre ella y sus otros dos hijos, Ioannes y $\mathrm{Kako}^{22}$. Al parecer la herencia de Iacobos se ha repartido en tres partes, una para la viuda y las otras dos para los hijos del matrimonio. Sin embargo, el hijo, Ioannes, mantiene también con Tapia otra batalla judicial, -paralela a la de Tsone- en relación a esta partición, alegando que su madre se ha apropiado de bienes que no le pertenecían ${ }^{23}$. También sabemos por $P$. Münch. I 9 que en el año 585 d.C., Tapia, aunque no se sabe la causa exacta, no se encuentra en Siene, sino que se ha trasladado a Antinópolis, $700 \mathrm{~km}$ Nilo abajo, junto a su hermano.

15. P. Lond. V 1731, 1s. 13-14.

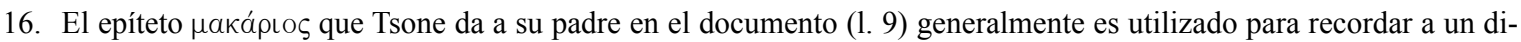
funto. O'Callaghan, 1964: 88-89; Bell, 1913: 166.

17. P. Lond. V 1731, 1. 18.

18. P. Lond. V 1731, 1s. 18-19.

19. P. Lond. V 1731, 1s. 19-20.

20. P. Lond. V 1731, 1s. 22-28.

21. Farber, 1990: 111-122.

22. P. Lond. V $1849+$ P. Münch. I 6. Es posible que Iacobos muriese sin testar sus bienes, y por ello se produce un problema a la hora de repartir la herencia entre la viuda y los hijos; Farber, 1990: 114 -117.

23. Parece repetirse la misma historia que había ocurrido unos años antes a la muerte de Díos, padre de Iacobos, que pudiendo también haber muerto intestado, esto podría haber generado el conflicto familiar entre la viuda y los hijos del matrimonio a causa de la herencia familiar, vid. P. Münch I 1; Faber, 1990: 112-113. La lucha entre Ioannes y Tapia se inicia en el año 583 d.C.: P. Lond. V $1849+$ P. Münch. I 6 - fecha en la que ya ha muerto Iacobos -, y finaliza en el año 594 d.C.: P. Münch. I 14. 
El conocimiento de los hechos ocurridos en el pasado de la familia, tanto lejano como más reciente, ayuda a la comprensión de la situación y desenlace de este conflicto. El asunto más importante es el divorcio entre Tapia y Menas, puesto que es la raíz de todo el problema. La ruptura parece haberse realizado por mutuo acuerdo entre los dos cónyuges. Una prueba de ello viene dada a través de la expresión que utiliza Tsone en la línea 11: "a causa de la energía del diablo y de Satán fuiste apartada de él", es decir: de Menas. Esta expresión para referirse a la causa de separación está constatada en Egipto desde el siglo IV d.C. ${ }^{24}$, parece tratarse de una expresión genérica, significando que se evita la culpabilidad de alguno de los cónyuges, para que no existan problemas posteriores para ninguno de los $\operatorname{dos}^{25}$.

En un divorcio por mutuo acuerdo entre la pareja, una mujer recuperaba su $\operatorname{dote}^{26} \mathrm{y}$ además podía recibir otras cantidades, como por ejemplo donaciones por parte del marido; éstas, si la mujer volvía a contraer matrimonio, debían transmitirse intactas, como habían sido recibidas, a los hijos de la unión precedente ${ }^{27}$. En estas cantidades es donde pueden situarse los cuatro sólidos de oro que reclama Tsone. Y posiblemente este dinero quedó reflejado en el acta del divorcio. No es extraño que se estipulara en el acta una cuantía para la manutención de la niña, puesto que en Egipto hay testimonios de hijos de matrimonios divorciados, que recibían la pensión alimenticia de su padre ${ }^{28}$.

La tutela de los hijos podía recaer en cualquiera de los cónyuges indistintamente; la decisión del cuidado de los hijos después de la ruptura pertenecía al ámbito privado, no al legal y en los papiros aparecen tanto al cuidado de la madre como al del padre ${ }^{29}$. En este caso, Tapia se hizo cargo de la niña en un primer momento, y aunque no sabemos cuanto tiempo estuvo con ella, debió de ser al menos un año. Esta suposición viene marcada porque cuando la separación era por consentimiento mutuo, la única pena que recibía la mujer era no poder volver a casarse en el plazo de un año ${ }^{30}$, y Tapia rechazó a su hija cuando se casó con Iacobos. Además Tsone pide todo el dinero que su padre dio, por lo tanto el tiempo de cuidado y manutención a cargo de la madre debió de ser breve.

\section{Estatus social y religioso de Aurelia Tsone}

La denominación de Aurelia indica el estatus social de Tsone, así como su origo, Siene. Generalmente son muy pocos los ascetas que aparecen en la documentación denominados con este título ${ }^{31}$. Entre las mujeres religiosas solamente existen otras dos que lo portan: dos hermanas, Teodora y Tauri, en P. Oxy. XLIV 3203 (400 d.C.) $)^{32}$.

24. En actas de divorcio conservadas el demonio aparece como la causa que explica la separación entre los dos cónyuges, Beaucamp, 1992 (vol II): 89-90. Una lista de las actas en: Beaucamp, 1992 (vol II): 139-140.

25. Justino II en la Nov. 140 del año 566 d.C. fundamenta la causa de la ruptura en los matrimonios por consentimiento mutuo, por sostener sufrimientos irracionales y desigualdades. Castello, 1984: 302-304.

26. CJ. $5,17,8$.

27. CTh. $3,8,2=$ CJ. $5,9,3$.

28. Till, 1938: 71-78.

29. P. Flor. I 93, P. Lond. V 1712 (569); P. Cair.Masp. II 67154, P. Cair.Masp. II 67155 (s. VI).

30. CJ. 5, 17, 9; Beaucamp, 1992 (vol. I): 227.

31. Según el estudio realizado recientemente por K. A. Worp, 2005: 145-152, únicamente el término Aurelio/a es aplicado a nueve monjes, entre los que está incluida Tsone, p. 150. A esta lista hay que añadir el P. Oxy. XLIV 3203 (Oxirrinco, 400 d.C.), que K. Worp no incluye, donde las dos religiosas también portan el título de Aurelia.

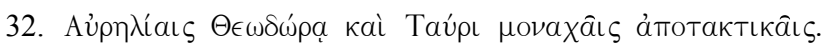


Según las investigaciones que se han realizado los títulos Aurelio o Aurelia designaban el estatus civil de una persona. Los Aurelii tenían ocupaciones fuera de asuntos oficiales o militares ${ }^{33}$. Esto hace pensar que Tsone fue denominada así en referencia a la condición social que hubiese tenido su padre, sin embargo tendría que haber abandonado el título cuando obtuvo su condición de religiosa.

Tapia y Kako llevan también esta denominación, la cual parece corresponder al oficio de Iacobos, puesto que el transporte fluvial se encuentra entre las ocupaciones que se denominan con el título ${ }^{34}$.

La onomástica familiar, basada en nombres coptos -Tapia, Menas, Tsone ${ }^{35}$, Kako, Patermucio...- induce a pensar que se trata de familias egipcias autóctonas.

La situación económica de Tsone durante su infancia tuvo que ser en cierta medida acomodada. Menas, cuando se hace cargo de ella, no reclama el supuesto dinero destinado a la manutención de la niña, y suponemos entonces, que él tiene los suficientes medios para poder alimentar, vestir y educar a su hija, y su educación parece estar dentro del ámbito urbano. La visión que se percibe es la de tratarse de una mujer in- dependiente y liberal, que tiene la capacidad y la libertad de actuar por su propio interés.

Una de las características más importantes de este papiro, y en este estudio la más importante, viene dada por la denominación de Tsone

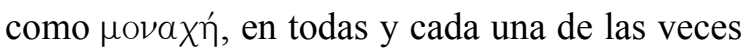
que aparece su nombre en el documento ${ }^{36}$. Este término es utilizado en el mundo griego clásico y helenístico tanto en su forma masculina:

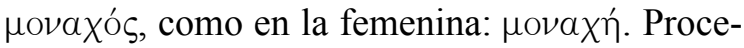
de del adjetivo griego $\mu$ óvos con el significado de "sólo, único", designa, entre otras acepciones, a un ser solitario y aislado de los otros y se aplica sobre todo a la condición de soltero o célibe. Durante el periodo anterior al surgimiento del monacato parece haber designado a los cristianos que renuncian a la vida conyugal, manteniéndose célibes. En la documentación papirológica el término es aplicado por primera vez al ambiente monástico en el año 324 d.C. en P. Coll. Youtie $77^{37}$, lo cual indicaría que el término era conocido por la sociedad para referirse a un asceta.

La primera mención en la literatura cristiana la hace Eusebio de Cesarea en el siglo IV d.C. en su Comentario a los Salmos ${ }^{38}$, al parecer escrito entre el 330 y 340 d.C. Para Eusebio los rovaxó son los cristianos que llevan una vida

33. Keenan. 1973: 51-55; Keenan, 1974: 295.

34. Keenan, 1973: 55.

[ 36 ] 35. Tsone en copto significa "la hermana". El nombre es utilizado en Siene en la misma época. En el mismo archivo en P. Lond. V 1724 y P. Münch. I 7 y 13 aparece otra mujer llamada igual, perteneciente a una familia vinculada en las transacciones económicas con la de Patermucio. Esta otra Aurelia Tsone es designada como hija de Apa Díos y Rachel y casada con un tal Isakos, el cual está implicado dentro de la trama entre Tapia y su hijo Ioannes. Cf. Tsone: SB Kopt. II 952; SB Kopt. II 1220; P. KRU 458; P. Lond.Copt. I 555; P. Lond. IV 576; O. Medin.HabuCopt. 47; P.Sarga 223; P. MoscowCopt. 196; O. CrumST 127; P. Mon.Epiph. 353.

36. Vid. n. 4.

37. Se trata de una petición al praepositus pagus, en la cual se hace mención a un rovaxó cllamado Isaac, que presta su ayuda a un vecino de la villa de Karanis, en el Fayum, en un altercado. Isidoro, el mencionado vecino, sufre daños en su huerto a causa del ganado colindante a su tierra, y posteriormente un asalto por parte de los dueños del mismo, quienes le golpean sin piedad. En ese momento recibió la ayuda del diácono Antonino y del monje Isaac, salvándose de morir golpeado.

38. $P G$ col. 689 B-C. 
solitaria, haciendo referencia a la continencia sexual. Atanasio de Alejandría utiliza el término en la Vida de San Antonio, poco tiempo después, hacia el 357 d.C., con el mismo significado pero ya aplicado al ambiente monástico.

En el año 585 d.C., finales del siglo VI d.C., el término está totalmente asentado dentro de la terminología monástica, y en todos los estudios en los que se hace referencia a este documento, como también consideramos en éste, no se ha dudado de reconocer a Tsone como una religiosa ${ }^{39}$.

En el papiro no está expresado el tipo concreto de vida ascética que llevaría Tsone y es difícil situarla dentro de una tipología determinada, puesto que debieron existir multitud de formas y variedades dentro de la vida monástica en Egipto $^{40}$. Ante tanta variedad, es preferible realizar una clasificación en torno a los contextos en los cuales se desarrollaba la vida monástica egipcia. Éstos se pueden dividir básicamente en tres: el desierto, el cenobio y la casa.

Para ubicar a Tsone dentro de uno de estos tres ambientes, la única definición que aparece en el

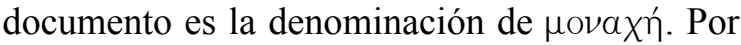
lo tanto, en primer lugar, tenemos que partir de la base de que este término en los papiros, no se refiere a ningún tipo concreto de ascetismo. De los diez papiros y ostraca que contienen la palabra en griego, únicamente en P. Cair.Masp. II 67141

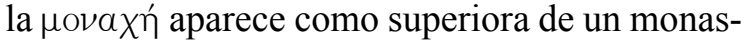
terio; en los otros nueve documentos no se deduce claramente un tipo concreto de ascetismo ${ }^{41}$.

Con los datos que hasta ahora hemos ido viendo es bastante improbable pensar que Tsone viviese en el desierto y tampoco se menciona su pertenencia a un monasterio ${ }^{42}$; por lo tanto, estos datos podrían inducir a pensar que practicase el ascetismo dentro del ámbito doméstico. La literatura monástica ofrece varios modelos de este tipo de ascetismo. El más antiguo de todos corresponde a pequeñas comunidades urbanas de vírgenes ${ }^{43}$; después aparece el modelo madrehija, que consistía en que una virgen consagrada habitaba en casa con su madre ${ }^{44}$; y también existe el tipo de asceta doméstica, que vivía en soledad en su propia casa llevando una vida de consagración a Cristo, bajo los principios básicos del monacato, y que se sustentaba con sus propios recursos ${ }^{45}$.

Este último modelo parece ajustarse a la vida que podría practicar Tsone. Seguramente, si su padre había muerto, ella vivía sola en su propia su casa y probablemente se sustentaba con sus propios bienes y recursos, de ahí que el dinero reclamado a su madre, quizá lo requiriera para este fin.

El monacato tiene una serie de características fundamentales que son comunes a todos los ti-

39. Bell, 1913: 168; Barison: 1938: 147; Wipszycka, 1997: 396; Rowlandson, 1998: 79-80; Geens, 2005 : 4.

40. Tradicionalmente se ha defendido la existencia de únicamente tres tipos de monjes, sin embargo es lógico pensar que debieron de existir una gran cantidad de tipos, como finalmente reconoció Goehring, 1999: 53-72.

41. PSI VI 698 (392); O. Douch. III 190 (s. IV); O. Douch. V 611 (s. IV); CPR V 26 (s. IV); P. Oxy. LVI 3862 (ss. IV-V); P. Oxy. XLIV 3203 (400); SB XVI 12525 (ss. V-VII); P. Cair.Masp. II 67138 (541-6); P. Lond. V 1731 (585).

42. En P. Lond. V 1724 (Siene, 578-82) perteneciente también al Archivo de Patermucio, se menciona un hombre llamado Ioannes pertenece al monasterio de Pampa, de la misma ciudad de Siene.

43. Atanasio de Alejandría en la Vita Antonii 3 menciona que Antonio dejó a su hermana en una de estas comunidades. Paladio en la Historia Lausiaca 4, también cuenta que las hermanas de Isidoro vivían en otra comunidad de vírgenes.

44. Paladio, Historia Lausiaca 31, 60; Pseudo Atanasio, c. 97; SB XVI 12620 (s. IV).

45. Paladio, Historia Lausiaca 63; Mosco, Pratum Spirituale 60. 
pos de vida ascética de cara a la sociedad: celibato, renuncia a los lazos familiares y a los bienes materiales, puesto que para practicar la ascesis era necesario desprenderse de todos los bienes terrenos ${ }^{46}$.

Sin embargo la única característica que parece seguir Tsone es el celibato -expresada a través del término monaché-, puesto que mantiene lazos familiares, ya que sigue vinculada a su familia a través del uso del término Aurelia. Tampoco prescinde de los bienes económicos, porque aunque supongamos que los sólidos reclamados eran para su sustento, parece más bien exigirlos simplemente como algo que le pertenece por derecho propio; y se trata de una sustanciosa cantidad si tenemos en cuenta que, la mitad de una casa en Siene en estos años, en los papiros del propio archivo, puede ser vendida por una cantidad similar de dinero ${ }^{47}$. No sabemos durante cuanto tiempo se prolongó esta disputa, pero sí sabemos que la lucha que mantiene su hermanastro Ioannes, al cual también Tapia al final debe entregarle la misma cantidad, dura once años; por lo tanto el valor de la suma parece ser importante ${ }^{48}$.

Como conclusión final podemos decir que P. Lond. V 1731 nos presenta en la última parte del siglo VI d.C., en la ciudad de Siene, a una religiosa, Aurelia Tsone, una mujer que tiene su propia independencia y que actúa libremente, sin ningún tipo de coacción. No abandona su posición social familiar y no renuncia a los bienes económicos, que considera le pertenecen por derecho propio y de forma directa. Su modo de vida podría corresponderse con el ascetismo doméstico, lo cual nos haría pensar que esta forma de vida aún se podría llevar a cabo, y no habría desaparecido en el siglo VI d.C. Sin embargo, ¿podemos considerar a Aurelia Tsone un modelo dentro de la vida ascética? La literatura ofrece una visión edificante y un tanto utópica del ideal monástico muy diferente a la realidad y hay que tener en cuenta, que estamos a finales del siglo VI d.C. y el monacato ha sufrido una evolución desde el siglo IV d.C., momento en el que este modelo de vida ascética tuvo su mayor auge.

46. Vergote, 1942: 329-345; Coquin, 1992: 15-24.

47. P. Münch. I 11 (586) y P. Münch. I 12 (590-1): la mitad de una casa es vendida por 5 sólidos de oro. Husson, 1990: 123-137.

48. P. Münch. I 14 (594). 


\section{Bibliografía}

BAgNALl, R. S.

1987 Church, State and Divorce in Late Roman Egypt, en: Selig, K.L.; Somerville, R. (eds.), Florilegium Columbianum: Essays in Honor of Paul Oskar Kristeller, New York: 41-61.

BARISON, P.

1938 Ricerche sui monasteri dell'Egitto bizantino ed arabo secondo documenti dei papiro greci, Aegyptus 18: 29-138.

BELL, H.I.

1913 Syene Papyri in the British Museum, Klio 13: 160-174.

BEAUCAMP, J.

1992 Le statut de la femme à Byzance (4e -7e siècle). Paris, 2 vols.

Castello, C.

1984 La Novella 140 di Giustino II e il malvagio

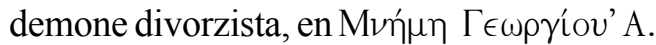
Пєтротоú $\backslash$ ov, Athènes, 1: 295-315.

Clackson, S.

1995 Four Coptic Papyri from the Pathermouthis Archive in the British Library, BASP 32: 97-116.

Coquin, R.-G.

1992 Évolution du Monachisme égyptien, Le Monde Copte 21-22 : 15-24.

EMMETT, A.

1982 Female ascetics in the greek papyri, en: XVI Internationaler Byzantinistenkongress, Akten II/2, Wien: 507-515.

FARBER, J. J.

1990 Family Financial Disputes in the Patermouthis Archive, BASP 27: 111-122.
FARber, J.; Porten, B.

1986 The Patermouthis Archive: A third look, BASP 23: 81-98.

GeEns, K.

2005 Archive of Flavius Patermouthis, son of Menas. Leuven.

GoEHring, J.

1999 Ascetics, society and the desert. Studies in early monasticism.

Harrisburg.

Husson, G.

1990 Houses in Syene in the Patermouthis Archive, BASP 27:123-137.

KeEnan, J.

1973 The Names Flavius and Aurelius as Status Designations, ZPE 11: 33-66.

1974 The names Flavius and Aurelius as status designations in Later Roman Egypt, ZPE 13: 283-304.

1990 Evidence for the Byzantine Army, BASP 27: 139-150.

MacCoull, L.

1990 Christianity at Syene/Elephantine/Philae, BASP 27: 151-162.

O'Callaghan, J.

1964 Epítetos de trato en la correspondencia cristiana del siglo VI, Studia Papyrologica 3.2: 79-105.

RowLANDSON, J.

1998 Women and society in Greek and Roman Egypt. A sourcebook. Cambridge.

RUSTAFJAELL, R.

1909 The Light of Egypt. London. 
TiLl, W. C.

WORP, K. A.

1938 Eine koptische Alimentenforderung, 2005 On the Aureliate of Clergy and Monks, $B S A C$ 4: 71-8.

ZPE 151: 145-152.

VERGOTE, J.

1942 L'Égypte, berceau du monachisme chrétien, CdÉ 33-34: 329-345. 


\section{Trabajos de Egiptología Papers on Ancient Egypt}

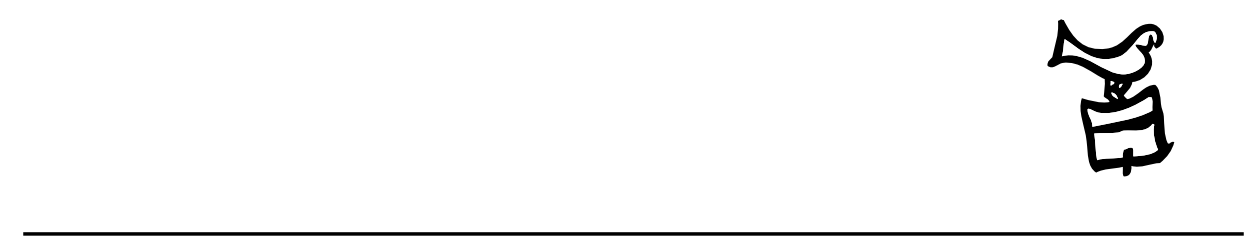

Número 5/1 2009 


\section{Actas \\ III Congreso Ibérico de Egiptología III Congresso Ibérico de Egiptologia}

Editores

Miguel Ángel Molinero Polo Covadonga Sevilla Cueva 


\title{
Editor
}

Miguel Ángel Molinero Polo

Universidad de La Laguna

\section{Consejo Editorial}

\author{
Antonio Pérez Largacha \\ Universidad de Castilla-La Mancha \\ José-R. Pérez-Accino \\ Birkbeck, Universidad de Londres \\ Covadonga Sevilla Cueva \\ Universidad Autónoma de Madrid
}

\section{Comité Científico}

Josep Cervelló i Autuori

Universitat Autònoma de Barcelona

$\mathrm{M}^{\mathrm{a}}$ José lópez Grande

Universidad Autónoma de Madrid

Josep Padró i Parcerisa

Universitat de Barcelona

$\mathrm{M}^{\mathrm{a}}$ Carmen Pérez Die

Museo Arqueológico Nacional, Madrid

Ester Pons Mellado

Museo Arqueológico Nacional, Madrid

José M. Serrano Delgado

Universidad de Sevilla

\section{Colaboradores Editoriales}

Linda Steynor

English editorial assistant

Hervé Mouriacoux

Assistant éditorial pour la langue française 
Trabajos de Egiptología está producida por Isfet. Egiptología e Historia c/ Blanco $1,2^{\circ}$

38400 Puerto de la Cruz

Tenerife-Islas Canarias

España

Maquetación: Proyecto Limón

(C) Autores de los artículos aparecidos

y Consejo Editorial de Trabajos de Egiptología - Papers on ancient Egypt

Depósito Legal: TF-2302-2009

ISSN: $1695-4750$

Imprime: Gráfica Los Majuelos, S.L.L.

imprenta@graficaslosmajuelos.com

Tfno.: 922311455 


\section{Comité Científico \\ III Congreso Ibérico de Egiptología III Congresso Ibérico de Egiptologia}

Miguel Á. Molinero Polo

Universidad de La Laguna

Presidente del Comité Organizador del III Congreso Ibérico de Egiptología

Miembro del Comité Organizador del I Encuentro de Egiptología

Josep Cervelló Autuori

Universitat Autònoma de Barcelona

Presidente del Comité Organizador del II Congreso Ibérico de Egiptologia

José Manuel Galán Allué

Consejo Superior de Investigaciones Cientificas

Director del Proyecto Djehuty, Luxor, Egipto

$\mathrm{M}^{\mathrm{a}}$ Helena Trindade Lopes

Universidad de Lisboa

Directora de la Misión Arqueológica Portuguesa en Menfis

Josep Padró i Parcerisa

Universitat de Barcelona

Director de la Misión Arqueológica de Oxirrinco

Antonio Pérez Largacha

Universidad de Castilla - La Mancha

Miembro del Comité Organizador del I Encuentro de Egiptología

José Ramón Pérez-Accino

Birkbeck College, University of London

Miembro del Comité Organizador del I Encuentro de Egiptología

$\mathrm{M}^{\mathrm{a}}$. Carmen Pérez Díe

Museo Arqueológico Nacional

Directora de la Misión Arqueológica Española en Heracleópolis Magna, Egipto

Covadonga Sevilla Cueva

Universidad Autónoma de Madrid

Miembro del Comité Organizador del I Encuentro de Egiptología 